Edith Cowan University

Research Online

Research outputs 2012

$1-1-2012$

\title{
Enhanced UV-Blue Response of Back Illuminated Deep Double Junction CMOS Compatible Photodiode Pixels; a Simulation Study of High Resolution Pixel Arrays
}

Paul Jansz

Steven Hinckley

Edith Cowan University

Follow this and additional works at: https://ro.ecu.edu.au/ecuworks2012

Part of the Engineering Commons

10.1109/COMMAD.2012.6472435

This is an Author's Accepted Manuscript of: Jansz, P. V., \& Hinckley, S. (2012). Enhanced UV-Blue Response of Back Illuminated Deep Double Junction CMOS Compatible Photodiode Pixels; a Simulation Study of High Resolution Pixel Arrays. Proceedings of 2012 Conference on Optoelectronic and Microelectronic Materials and Devices (COMMAD). (pp. 211-212). Melbourne, Australia. IEEE. Available here (C) 2012 IEEE. Personal use of this material is permitted. Permission from IEEE must be obtained for all other uses, in any current or future media, including reprinting/republishing this material for advertising or promotional purposes, creating new collective works, for resale or redistribution to servers or lists, or reuse of any copyrighted component of this work in other works.

This Conference Proceeding is posted at Research Online.

https://ro.ecu.edu.au/ecuworks2012/192 


\title{
Enhanced UV-Blue Response of Back Illuminated Deep Double Junction CMOS Compatible Photodiode Pixels; a Simulation Study of High Resolution Pixel Arrays
}

\author{
Paul V. Jansz* and Steven Hinckley \\ Centre for Communications Engineering Research, Edith Cowan University, WA 6027, Australia \\ ${ }^{*}$ Corresponding author: Email p.jansz@ecu.edu.au
}

\begin{abstract}
Simulated backwall U/V blue illuminated double junction photodiode (DJPD) pixels demonstrate excellent crosstalk and sensitivity when substrate, guard and image wells were doped more highly and biased minimally. This configuration minimized each guard and image well depletion region (SCR) width, so that the image SCR could be presented as close as possible to the back wall without the guard and image SCRs overlapping between and within pixels.
\end{abstract}

1 Introduction: There is a need to develop efficient, low-cost UV-blue enhanced imaging arrays with high resolution and fast response times for many different applications. These applications include flame and fire detection [1], astronomy [2], nuclear scintillation detectors [3], and electrophoretic band detection [4]. Back illuminated arrays also have application in particle physics, plasma spectroscopy and astrophysics [5].

The development of UV-Blue sensitive arrays has led to the investigation of shallow polywell-stacked gradient polyhomojunction (StaG) hybrid geometries and deep implanted single junction well geometries [5]. Backwall illumination is of particular interest, due to increased fill factor and the ability to tailor individual photodiodes (PD) to a specific wavelength band. The StaG architecture has demonstrated improved sensitivity and crosstalk suppression. However, for the backwall illumination mode to have greater response resolution than frontwall illumination for UV application, the photocarriers need to be captured exclusively and thoroughly by each pixel, which is not possible by backwall depletion alone. Previous deep implanted-well single junction PD simulation studies, though show

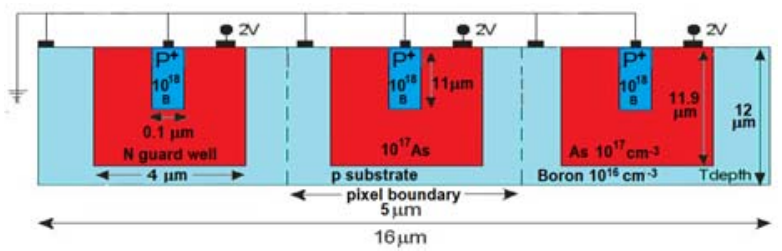

Fig. 1. Tripixel DJPD configuration. Dimensions vary depending on trial.

ing improvement in sensitivity (maximum QE) and crosstalk, especially for the UVBlue (400-450) [5], indicate that crosstalk still needs significant reduction, with no sensitivity loss.

2 Method: The simulated [5] geometry (Fig.1) used nested etch-epitaxially grown wells that allowed for outward Gaussian diffusion. The $\mathrm{P}^{+} \mathrm{N}$ and NP junctions were designated image and guard junctions. Normal 400 to $800 \mathrm{~nm}$ monochromatic total pixel laser illumination was used. Crosstalk being the quantum efficiency (QE) at the $\mathrm{P}^{+}$electrode of the central pixel for total illumination of the adjacent left pixel. Similarly, sensitivity was the QE at the same image electrode upon total normal illumination of the central pixel.

3 Results: Fig. 2 and 3 show crosstalk and sensitivity dependence on guard pitch, respectively. Fig. 4 and 5 show crosstalk and sensitivity dependence on image well depth.

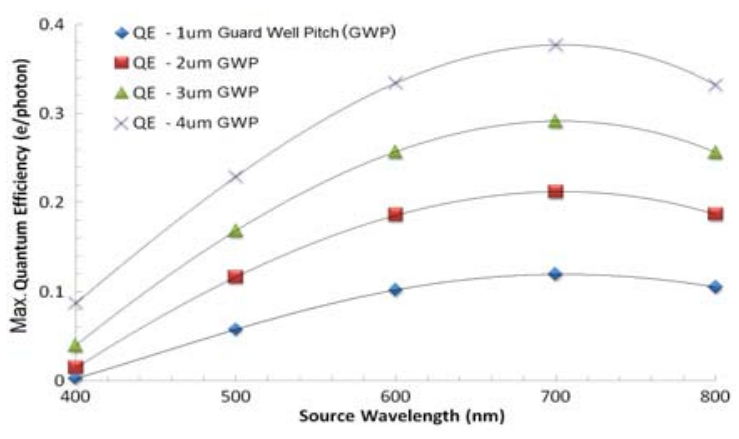

Fig. 2. Sensitivity dependence on guard well pitch. Substrate, guard and image well depth are $12 \mu \mathrm{m}, 11.9 \mu \mathrm{m}$, and $11 \mu \mathrm{m}$ respectively. Image well pitch is $0.1 \mu \mathrm{m}$. 


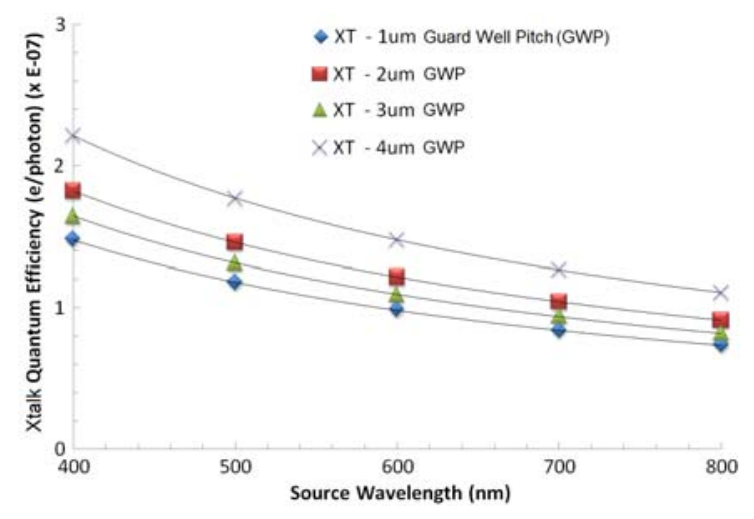

Fig. 3. Crosstalk dependence on guard well pitch. Substrate, guard and image well depth are $12 \mu \mathrm{m}, 11.9 \mu \mathrm{m}$, and $11 \mu \mathrm{m}$ respectively. Image well pitch is $0.1 \mu \mathrm{m}$.

All crosstalk results (Fig. 3 \& 5) for back illumination are significantly reduced. The second outer guard well's SCR captures the crosstalk carriers exclusively, leaving only the photocarriers unique to each pixel being captured by each pixels image-electrode. This also applies to front illumination, however, the immediacy of both SCRs to front illumination results in excellent crosstalk suppression and sensitivity for all CMOS sensitive wavelengths (400-1000nm), with loss of wavelength discrimination. Back illumination DJPD sensitivity is wavelength discriminatory (Fig. 2 \& 4) due to its wavelength dependent absorption profile in proximity to the double junction cross-section. This is shown by UV/Blue sensitivity being best when the guard well is widest (Fig. 2) and the image well is deepest (Fig. 4), because UV /blue absorption occurs much closer to the back wall than longer wavelengths.

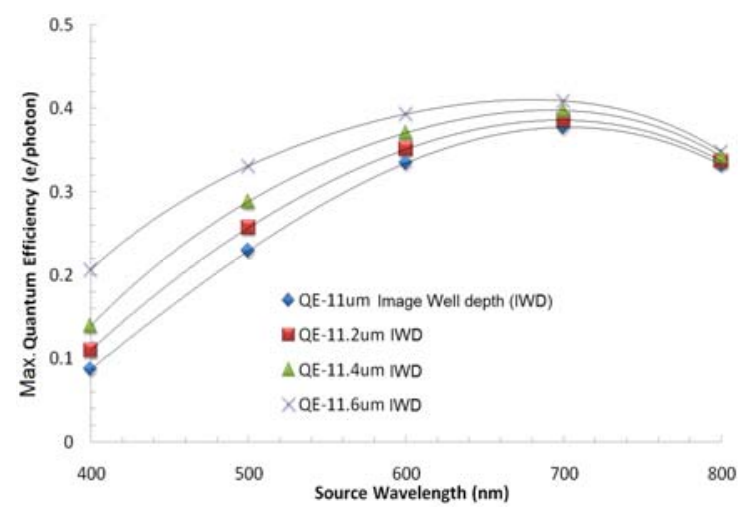

Fig. 4. Sensitivity dependence on image well depth. Substrate and guard well depth are $12 \mu \mathrm{m}$ and $11.9 \mu \mathrm{m}$ respectively. Guard and Image well pitch are 4 and $0.1 \mu \mathrm{m}$ respectively.

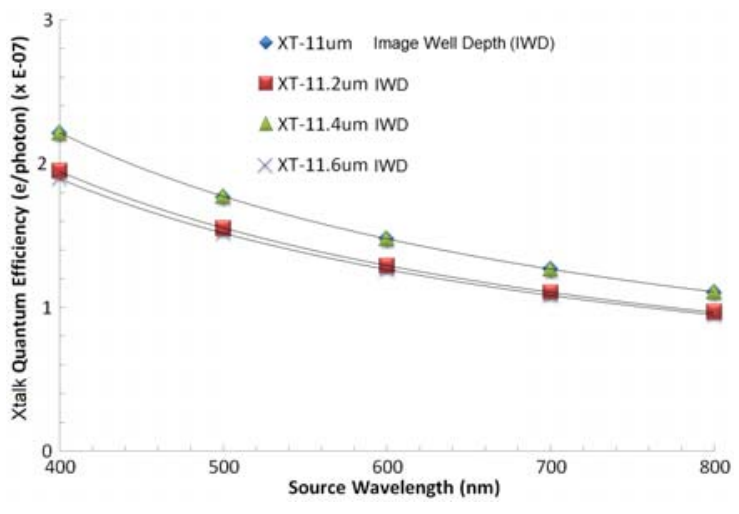

Fig. 5. Crosstalk dependence on image well depth. Substrate and guard well depth are $12 \mu \mathrm{m}$ and $11.9 \mu \mathrm{m}$, respectively. Guard and Image well pitch are $4 \mu \mathrm{m}$ and $0.1 \mu \mathrm{m}$, respectively.

4 Future Work: The benefits of wider and multiple image wells, will be explored to the UV limits of CMOS. Deep implantation of Image wells will be assessed as implantation may be fabricationally less intensive then the deep-etch-epitaxial procedure, simulated for guard and image wells in this research. Ultimately, the possible colour discriminating ability of DJPD pixel architectures needs investigation.

5 Conclusion: Considerable reductions in crosstalk can be achieved with a deep DJPD CMOS array structure, while maintaining high sensitivity level for backwall $\mathrm{UV} /$ blue illuminated high resolution pixels.

\section{References}

[1] D. Starokov, C. Boney, R. Pillai, and A. Bensaoula, "Dual-band UV/IR optical sensors for fire and flame detection and target recognition," in Proc. IEEE Sensors for Industry Conference, pp. 36-40, 2004.

[2] M. P. Ulmer, M. Razeghi, and E. Bigan, "Ultraviolet detectors for astrophysics: Present and future," in Proc. SPIE, vol. 2397, pp. 210217, 1995.

[3] D. Caputo, G. de Cesare, G., A. Nascetti, and M. Tucci, "Innovative window layer for amorphous silicon/amorphous silicon carbide UV sensor," Journal of Non-Crystalline Solids, vol. 352, pp. 1818-1821, 2006.

[4] Z. Xu, T. Nishine, A. Arai, and T. Hirokawa, "Performance of electrokinetic supercharging for high-sensitivity detection of DNA fragments in chip gel electrophoresis," Electrophoresis, vol. 25, pp. 3875-3881, 2004.

[5] P. V. Jansz and S. Hinckley, "Simulation of crosstalk and quantum efficiency in highresolution UV-blue imaging arrays," in Proc SPIE, vol. 8204, pp. 82042X, December 2011. 